\title{
Management of venous thrombosis in the pediatric patient
}

This article was published in the following Dove Press journal:

Pediatric Health, Medicine and Therapeutics

21 July 2015

Number of times this article has been viewed

\author{
Vlad C Radulescu \\ Department of Pediatrics, University \\ of Kentucky, Lexington, KY, USA
}

\begin{abstract}
The incidence of venous thromboembolism in children has increased significantly over the past decade. The evaluation and management of the child with venous thromboembolism, while based on the adult experience, has its own particularities dictated by the differences in the hemostatic system of the newborn and child. The current review addresses the evaluation of pediatric patient with thrombosis as well as the established and emerging treatment interventions.
\end{abstract}

Keywords: venous thromboembolism, children, anticoagulant therapy, thrombolytic therapy

\section{Introduction}

Deep venous thrombosis (DVT) occurs in the inferior and superior vena cava, their major tributaries, the deep veins of the extremities, the splanchnic veins, as well as the cerebral venous sinuses. The DVT may lead to acute venous obstruction with possible organ compromise, pulmonary embolism and, on long term, to venous insufficiency and post-thrombotic syndrome (PTS).

The incidence of venous thromboembolism (VTE) increases with age. In the pediatric population (0-18 years) the incidence is approximately $0.7-2.1$ cases per 100,000 children, ${ }^{1-3}$ while in the adult population the incidence is $100-150$ per $100,000 .{ }^{4,5}$

The VTE incidence in children has two peaks, one in infancy and another one in adolescence. Several studies showed a significant increase in the VTE incidence in children and adolescents over the past 2 decades. ${ }^{6,7}$ The trend may be related to complex therapies that increase the survival of very sick children but also carry the risk of iatrogenic complications, improved imaging modalities, increased VTE awareness among providers, and possibly changes in the prevalence of conditions associated with VTE, like obesity. ${ }^{8}$ While pediatric VTE becomes more prevalent, it is still an uncommon condition compared to the adults. Most of the clinical data used in treatment decisions come from adult studies. Sometimes the adult data cannot be extrapolated to children given age-related physiological difference in the hemostatic system and particular responses to anticoagulant and thrombolytic medications. ${ }^{9}$

\section{Pathophysiology}

Hemostasis is a tightly regulated process aimed at maintaining the integrity of the vascular system by preventing leaks. The system is kept in balance through the interaction of pro-coagulant factors on one side, and anticoagulant and fibrinolytic factors on the other. The DVT occurs when the hemostatic equilibrium is tilted toward clot 
formation. Virtually all the factors that tilt this balance toward thrombosis act through three main mechanisms, as recognized by the German physician Virchow more than 1 century ago: venostasis, endothelial damage, and hypercoagulability.

\section{Etiology}

There are numerous risk factors that contribute to the development of an abnormal thrombus. Identification of the causative factors of VTE as well as determining the reversibility of these factors is very important in planning the therapy, in terms of length and intensity of the interventions, and assessment of the risk of recurrence of the thrombosis (Table 1).

Some of the causative factors are clinically apparent and potentially reversible: the presence of central venous lines, trauma, major surgery followed by bedrest, immobilization, infection (cellulitis, osteomyelitis, phlebitis), cancer chemotherapy (L-asparaginase), inflammatory bowel disease, nephrotic syndrome, obesity, high estrogenic states (pregnancy, use of estrogen-containing hormonal contraceptives), dehydration, and total parenteral nutrition. The majority of the pediatric patients with VTE have at least one of these risk factors.

Other risk factors may persist for a long period of time, even lifelong, though they are not inherited. One group

Table I Risk factors for pediatric venous thromboembolism (VTE)

\begin{tabular}{l}
\hline Temporary/potentially reversible \\
Central venous catheters \\
Trauma \\
Major surgery \\
Immobilization \\
Infection (sepsis, cellulitis, osteomyelitis) \\
Inflammatory bowel disease \\
Nephrotic syndrome \\
Obesity \\
Pregnancy \\
Estrogen containing contraceptives \\
Dehydration \\
Total parenteral nutrition \\
Cancer \\
Cancer chemotherapy \\
Long-lasting \\
Antiphospholipid antibodies \\
Congenital heart disease \\
Vascular/anatomic anomalies \\
Inherited risk factors \\
Antithrombin deficiency \\
Protein C deficiency \\
Protein S deficiency \\
Factor V Leiden \\
Prothrombin gene 202I0A mutation \\
Elevated homocysteine \\
Family history of VTE \\
\hline
\end{tabular}

includes the presence of antiphospholipid antibodies: anticardiolipin antibodies, anti-beta 2 GP1 antibodies, and lupus anticoagulant. Another group includes the congenital heart malformations and other anatomic anomalies that produce venostasis in the tributaries. May-Thurner syndrome refers to left iliac vein compression between the right iliac artery crossing anteriorly and the lumbar vertebrae. Paget-Schroetter syndrome occurs in subjects with a narrow costoclavicular space. Upon vigorous, repetitive, movements of the arm, these subjects develop micro-trauma and subsequent thrombosis in the subclavian vein.

Lastly, there is the group of inherited risk factors that lead to a lifelong tendency toward hypercoagulability, commonly referred to as inherited thrombophilia. These include: antithrombin deficiency, Protein C deficiency, Protein S deficiency, factor V Leiden, prothrombin gene 20210A mutation, elevated homocysteine levels secondary to the MTHFR mutation. ${ }^{10} \mathrm{~A}$ family history positive for the presence of venous thrombotic events represents another inherited risk factor for thrombosis especially if it involves close relatives, who were young at the time of thrombosis, and if the VTE was unprovoked; a history of repeated fetal loss in female relatives may suggest an inherited hypercoagulable state.

Unlike VTE in older adults, in children an inherited hypercoagulable trait is rarely the only risk factor for DVT, but it does play a role in tilting the hemostatic balance and potentiating the acquired risk factors described earlier.

\section{Evaluation of thrombosis Imaging studies}

The imaging modality of choice varies according to the location of the thrombosis. For extremity veins, the color flow Doppler ultrasound is the preferred study given it is not invasive, does not involve radiation, does not require sedation, and offers reliable information regarding the blood flow through the vessels. The Doppler ultrasound may be used for the evaluation of the portal and renal veins, but it is less reliable in evaluation of the inferior vena cava (IVC) and its main tributaries. The right atrium and the proximal portions of the superior vena cava and IVC can be visualized through echocardiography. Computerized tomography (CT) angiography and magnetic resonance venogram can be employed to visualize the venous blood flow in the cerebral venous sinuses and the deep veins in the thorax and abdomen. The contrast venogram may be useful for imaging of central catheter related thrombosis. Pulmonary embolism, in children, is best evaluated with CT angiography. 


\section{Laboratory evaluation}

There are no global tests for hemostasis that are validated for evaluation of the hypercoagulable tendency in VTE. The thrombelastogram and the thrombin generation assays are global tests of hemostasis that offer information about both the bleeding and clotting tendencies; while the thrombelastogram has been used in the management of extracorporeal circuits, neither of the two has been used in the management of VTE. ${ }^{11}$

The measurement of the levels of AT, Protein C, and Protein S, or the identification of factor V Leiden or prothrombin gene mutation only gives information about one particular component of the hemostatic system but does not allow the quantification of the overall thrombotic tendency which results from the interplay between inherited and acquired factors.

When evaluating the AT, Protein $\mathrm{C}$, and Protein S, one has to take into account different factors that may affect their levels. All three proteins have physiologically low levels in the first 3-6 months of life, and they may be consumed, thus falsely low, during the acute thrombotic event. The AT level may be influenced by heparin therapy, whereas the Protein C and Protein S activity may be falsely low during therapy with vitamin $\mathrm{K}$ antagonists. Last but not least, some of the clotting based assays for Protein $\mathrm{C}$ and Protein S may be influenced by the factor V Leiden mutation, factor VIII levels, or the presence of the lupus anticoagulant. ${ }^{12}$

The results of the thrombophilia evaluation do not influence the immediate management of the VTE. The exceptions are the extremely rare homozygous Protein $\mathrm{C}$ or Protein S deficiency; these patients, will present in the neonatal period with extensive thrombosis and necrotic skin lesions of purpura fulminans; a diagnosis of severe Protein $\mathrm{C}$ or Protein $\mathrm{S}$ deficiency may prompt replacement therapy in addition to the anticoagulant therapy. The thrombophilia evaluation may provide information about the risk of recurrence and guidance toward the preventative intervention that should be recommended for the affected patient.

There are no clear guidelines regarding which VTE patients should be evaluated for thrombophilia. Most authors would agree that all neonates with non-catheter related DVT and adolescents with spontaneous, non-provoked VTE should be evaluated, along with children with VTE who have a family history of thrombosis or a close relative diagnosed with inherited thrombophilia. ${ }^{13}$ Thrombophilia testing should be considered in children with recurrent thrombosis. Testing of asymptomatic children is controversial and it is not supported by epidemiologic data.
The $\mathrm{D}$-dimer level is a measurement of fibrin production and degradation. It has been used in adults in the diagnostic algorithms of pulmonary embolism. The pediatric normal ranges are age dependent. ${ }^{14}$ In children with suspected pulmonary embolism, a normal D-dimer level does not have the same negative predictive value as in adults: up to $15 \%$ of children with pulmonary embolism (PE) have a normal D-dimer level. ${ }^{15,16}$

For adults with VTE the persistently elevated D-dimer and factor VIII levels several months after diagnosis are predictive of DVT recurrence. There is limited pediatric data showing that elevated D-dimer and factor VIII levels 3-6 months after diagnosis are associated with DVT recurrence. ${ }^{17,18}$

\section{Treatment}

The goals of the VTE therapy are to prevent clot extension, prevent embolism, restore venous patency, limit the longterm sequelae, and reduce the risk of recurrence. These goals are achieved by rebalancing the hemostatic system with anticoagulant and thrombolytic therapy and, when possible, reversal of the causative factors.

There are several factors to be taken into account when choosing the initial therapy which will be discussed now.

\section{The urgency of the intervention}

Certain presentations, like a large pulmonary embolism causing hemodynamic instability or a large venous obstruction risking organ or limb compromise require a rapid resolution of the thrombus. Thrombolysis should be considered in such situations rather than anticoagulation only.

\section{The risk of long-term sequelae}

Large, obstructive thrombi in the lower extremities and IVC are more likely to cause PTS, manifested by pain, swelling, and skin abnormalities. There are several adult and pediatric series suggesting that thrombolysis is superior to anticoagulation in preventing the development of PTS. ${ }^{19,20}$

\section{The risk of the intervention}

Both anticoagulation and thrombolysis have a risk of bleeding; the risk would be higher for patients with ongoing bleeding (intracranial hemorrhage, gastrointestinal hemorrhage, recent surgery, or trauma) or preexisting bleeding tendencies (thrombocytopenia, coagulopathies, prematurity). 


\section{The level of expertise and proficiency of the medical team}

Interventions like catheter-directed thrombolysis often represent a team effort involving an interventional radiologist or cardiologist, a hematologist, and an intensive care specialist; given the relatively low patient volumes, such a team may be difficult to assemble and maintain its proficiency, especially for the care of neonates and young children.

\section{Anticoagulant therapy}

The anticoagulant therapy reduces the risk of thrombus extension and embolism while allowing the natural fibrinolytic mechanism to gradually reduce the size of the clot.

\section{Duration}

The duration of anticoagulant therapy is mainly based on adult studies. Several factors should be taken into consideration; if the causative factor can be identified and is reversible one may consider a shorter course; the resolution of the thrombus, and normalization of D-dimer and factor VIII levels have been associated with a lower risk of recurrence, thus may support a shorter duration of anticoagulation..$^{21} \mathrm{~A}$ recurrent VTE, especially if the cause is elusive would warrant longterm anticoagulation.

For the first VTE event the American College of Chest Physicians (AACP) guidelines ${ }^{22}$ recommend anticoagulation with heparin or low molecular weight heparin (LMWH) followed by a vitamin $\mathrm{K}$ antagonist for 3 months if the causative factor can be identified and resolved, and 6-12 months for an unprovoked VTE. For recurrent VTEs the recommended duration of anticoagulation is 3 months for provoked VTE if the causative factor can be resolved, and lifelong for idiopathic, unprovoked VTE.

\section{Anticoagulant agents}

Unfractionated heparin acts predominantly by enhancing the inhibitory action of AT on thrombin, factors Xa, IXa, XIa; it may be administered subcutaneously or intravenously. It has a relatively short half-life: $30-60$ minutes, with intravenous administration. The anti-coagulant activity of heparin may be monitored using the activated partial thromboplastin time (aPTT) or the anti-activated factor X activity (anti-Xa); the therapeutic range is extrapolated from adult VTE studies. Given that the aPTT has higher normal values in neonates and infants, it is difficult to define a therapeutic range for aPTT. The anti-Xa is considered a more reliable test for this age group. The generally accepted therapeutic range is $0.35-0.7 \mathrm{U} / \mathrm{mL}$. Newborns have physiologically lower AT levels and thus require higher heparin doses. The starting therapeutic dose for heparin, when used as a continuous infusion, is $28 \mathrm{U} / \mathrm{kg}$ for neonates and $18-20 \mathrm{U} / \mathrm{kg}$ for children older than 1 year. The dose should be titrated to the desired level of anticoagulation. The use of bolus infusion of 75-100 $\mathrm{U} / \mathrm{kg}$ shortens the time to achieve the therapeutic range but may increase the risk of bleeding especially in premature newborns. The main risk associated with heparin use is bleeding, with estimates of major bleeding varying widely between $1.5 \%$ and $24 \%,{ }^{23}$ depending on the population and the clinical setting studied. The risk of developing heparin induced thrombocytopenia (HIT) is relatively low, up to $1 \%-2.3 \% .^{24,25}$ The risk of osteoporosis with long-term use of unfractionated heparin is unknown, with only a few cases reported.

LMWHs act through the AT pathway, and have a stronger inhibitory effect on the Xa as compared to unfractionated heparin and secondary anti-IIa effect. Enoxaparin, the most commonly used LMWH in children, has a longer half-life than unfractionated heparin - approximately 12 hours, and has a more predictable dose-response relationship. It shows predominant renal excretion, thus it should be used with caution in individuals with renal insufficiency.

The anticoagulant activity of LMWH is measured by the anti-Xa activity. The therapeutic range is between 0.5 and $1 \mathrm{U} / \mathrm{mL}$, measured 3-5 hours after subcutaneous (sq) administration. Newborns require larger doses per kilogram. For enoxaparin the starting therapeutic dose is $1.5 \mathrm{mg} / \mathrm{kg}$ every 12 hours sq if the patient is younger than 2 months, and $1 \mathrm{mg} / \mathrm{kg}$ above this age. Once the therapeutic levels are achieved, periodic monitoring of anti-Xa activity is recommended, especially in young infants, who experience rapid weight gain and changing LMWH requirements per kilogram of body weight.

The main risk of LMWH therapy is bleeding, with different authors reporting rates of major bleeding between $2.9 \%$ and $5 \%$. The anticoagulant activity of LMWH can be partially reversed using protamine. Protamine is thought to reverse the anti-IIa activity but not the anti-Xa activity. Osteoporosis and HIT are thought to be rare complications of LMWH therapy, though there are no data regarding their incidence in pediatrics.

Vitamin $\mathrm{K}$ antagonists act through inhibition of vitamin $\mathrm{K}$ dependent, post-translational carboxylation of coagulation factors II, VII, IX, and X. The most commonly used agent is warfarin. The anticoagulant activity is measured by the prothrombin time (PT)/international normalized ratio (INR). The PT/INR results from tests performed using point-of-care monitors are comparable in terms of accuracy and reliability 
with INR performed on venous blood in clinical laboratories. ${ }^{26}$ The therapeutic range for most indications, extrapolated from adult studies, is an INR of 2-3. Patients with artificial heart valves require more intense anticoagulation. ${ }^{27}$ Warfarin is, in general, not used in newborns and young infants because of the difficulty of keeping the patient in the therapeutic range. Newborns have physiologically low levels of vitamin $\mathrm{K}$ dependent factors and variable intake of vitamin $\mathrm{K}$ (low in breast milk, high in formula and the total parenteral infusions); the blood sampling for INR monitoring is often difficult and there is no liquid formulation for warfarin. The main risk of warfarin therapy is bleeding. The risk of major bleeding varies between different reports, being $0.05 \% 0^{28}-12.2 \%{ }^{29}$ per patient per year, though many reports place the risk at approximately 3\% per patient per year. ${ }^{30}$ An important factor in reducing the risk of bleeding seems to be the education of the patient and family in managing this medication. The effect of warfarin may be reversed in case of an extremely high INR or active bleeding using vitamin $\mathrm{K}$, fresh frozen plasma infusions, prothrombin protein concentrates, or recombinant activated factor VII. Warfarin is teratogenic, thus adolescent females should receive appropriate counseling.

Upon initiation of therapy, warfarin is administered along with heparin or LMWH for 4-6 days, until a therapeutic INR is achieved. This approach prevents the development of a transient hypercoagulable state through the inhibition of Protein $\mathrm{C}$ and Protein S post-translational carboxylation.

\section{Newer anticoagulants}

Argatroban is a direct thrombin inhibitor approved for pediatric use in the United States for treatment of patients with HIT. It is given as a continuous intravenous infusion, and its anticoagulant activity is monitored with the aPTT, with a goal of aPTT of 1.5-3 times the normal, measured 2 hours after a steady IV infusion. ${ }^{31}$ Argatroban has hepatic clearance, and thus should be used with caution in critical patients with hepatic dysfunction.

The direct thrombin inhibitor dabigatran has been approved for adult use, but the pediatric experience is limited to small case series; there are ongoing pediatric clinical trials. $^{32}$

Fondaparinux is a direct factor Xa inhibitor, which does not interact with the platelets, thus does not trigger HIT. It is administered at daily intervals by subcutaneous route. Its use has been studied in children, ${ }^{33}$ though it is not approved for pediatric use in the US.

Direct anti-Xa inhibitors rivaroxaban and apixaban are widely used in adults, whereas the pediatric experience is limited to small case series; there are several ongoing clinical trials.

Dalteparin, an LMWH used in adults, has been studied in children; a standardized age-based dosing study successfully achieved anti-Xa levels, with a favorable safety profile. ${ }^{34}$

\section{Thrombolysis}

The goal of the thrombolysis is to relieve the vascular obstruction produced by the clot in a short period of time by activating the fibrinolytic system. It is used in life- or limbthreatening thrombosis, when time is of the essence. There are reports of the use of thrombolysis in venous thrombosis with the goal of reducing the incidence of PTS.

The agent of choice in the pediatric population is the tissue plasminogen inhibitor activator (tPA). tPA may be administered systemically, or via a catheter, at the site of thrombosis. In systemic thrombolysis it may be administered in doses of $0.1-0.6 \mathrm{mg} / \mathrm{kg}$ for 6 hours, as suggested in the AACP guidelines ${ }^{22}$ though alternative regimens involving lower doses and longer exposure times $(0.01-0.06 \mathrm{mg} / \mathrm{kg} / \mathrm{hr}$ for 4-48 hours) have been proposed as well. ${ }^{35}$ The catheterdirected thrombolysis uses lower doses of thrombolytic agent delivered in the proximity of the clot for various lengths of time. ${ }^{36,37}$ Anticoagulation with low-dose heparin may be used concurrently with the thrombolytic agent with the rationale of stopping the clot propagation. ${ }^{38}$

The main risk associated with thrombolysis is bleeding. The risk of a major bleed, defined as a bleed that requires a blood transfusion or other medical intervention, varies significantly between different reports. This is not surprising giving the absence of large studies and the range of patient populations and thrombolytic regimens used in various case series. ${ }^{39}$ The results of a meta-analysis which included 413 cases of children receiving thrombolytic therapy placed the incidence of major hemorrhage at $15 \% .{ }^{39}$ In order to decrease the risk of bleeding, a baseline evaluation prior to the start of thrombolysis should include, at a minimum, a platelet count, fibrinogen level, PT, PTT and, in newborns, a head ultrasound to rule out a preexisting intracranial hemorrhage.

The contraindications for thrombolysis according to the International Society of Thrombosis and Haemostasis guidelines include: prematurity (less than 32 weeks gestation), recent surgery or hemorrhage, recent severe hypoxic event, sepsis, seizures within the previous 2 days, intractable thrombocytopenia, or hypofibrinogenemia. ${ }^{38}$ It should be noted that no randomized trials have been conducted in children on the use of thrombolytic therapy, and there is a relatively small number of children treated with institutional 
regimens, with different doses and duration of therapy; thus, the benefits and risks of this intervention have to be weighed in each individual case.

\section{IVC filters}

IVC filters have been used in adults since the 1970s. There are several reports of their use in children. ${ }^{40,41}$ The most common reason for placement of an IVC filter is a contraindication for anticoagulation in a patient at risk for pulmonary embolism. Other indications include recurrent DVT despite anticoagulation therapy and PE prevention during endovascular thrombolysis. The filters may be retrievable or permanent, and their use is generally limited to children heavier than 10 $\mathrm{kg}$. The main risks include the extension of the thrombus to the filter, thrombus formation within the filter, perforation of the IVC, or migration of the filter. ${ }^{42}$ The long-term risks of IVC filters in pediatric patients are unknown. The IVC filter should be removed as soon as the risk of PE decreases; different device manufacturers recommend the removal of the filters within weeks to months after placement.

\section{Thrombectomy}

The surgical removal of thrombi is reserved for life-threatening VTE such as large pulmonary embolism or occlusion of vascular shunts after cardiac surgery. There are no specific guidelines, and the procedures have a significant rate of complications, including recurrent thrombosis and vascular damage; therefore, the risks and the benefits should be carefully evaluated in a case-by-case basis.

\section{VTE prophylaxis}

VTE is a relatively common complication in hospitalized adults and one of the main preventable causes of hospital death. With increasing incidence of DVT in the pediatric population, the issue of VTE prophylaxis in hospitalized children has gained increasing attention. Although there is no consensus, institutional ${ }^{43,44}$ or regional ${ }^{45}$ guidelines recommend VTE prophylaxis in children who have several risk factors for thrombosis, typically immobilization in addition to at least one more risk factor. Age seems to play a role in the decision, adolescents being more likely to receive prophylaxis, since their risk profile resembles that of the young adult population.

VTE prophylaxis combines physical as well as pharmacologic methods. The physical methods like sequential compression devices or compression stockings aim to reduce the venostasis associated with bedrest. The most commonly used anticoagulants for short-term prophylaxis are the LMWHs in doses that are typically half of that used in VTE treatment regimens (for enoxaparin $0.5 \mathrm{mg} / \mathrm{kg}$ every 12 hours sq or 30-40 mg sq for larger, adult-sized adolescents).

\section{Specific circumstances Newborns}

The hemostatic system of the newborn has its own particularities: most of the clotting and antithrombotic factors as well as the components of the fibrinolytic system are produced in lower quantities than older children and adults. ${ }^{9}$ The exact implications for the hemostatic balance are unclear, though normal, healthy, newborns rarely develop thrombosis even if they are carriers of inherited thrombophilic traits. Neonatal thrombosis occurs in approximately $90 \%$ of cases in the context of the presence of a central venous catheter and the condition that prompted the catheter placement: neonatal asphyxia, sepsis, dehydration, maternal diabetes, and congenital heart disease. Unprovoked thromboses are rare. The most common unprovoked neonatal DVT is renal vein thrombosis. This condition is frequently associated with inherited thrombophilia. ${ }^{46}$

Newborns require higher doses of heparin per kilogram in order to acquire a certain level of anticoagulation due to the physiologically low levels of AT. Newborns have low plasminogen inhibitor levels; thus, when using tPA in thrombolytic therapy they require fresh frozen plasma for plasminogen inhibitor supplementation. Premature newborns are at risk of intraventricular hemorrhage. Baseline brain imaging, prior to the start of anticoagulant or thrombolytic therapy, is very important in assessing the risk versus benefit of the therapeutic intervention.

\section{Central venous catheters}

A central venous catheter is present in approximately $90 \%$ of newborns and more than $50 \%$ of children who develop DVT, ${ }^{47}$ and thus is considered to be one of the main risk factors for DVT in children. The catheter thrombosis may manifest as a fibrin sheath around the catheter leading to catheter malfunction or a larger thrombus that adheres to both the catheter and the venous wall and may produce vascular obstruction, pulmonary embolism, or even paradoxical embolism and stroke in patients with a right to left or bidirectional shunt. The presence of the thrombus increases the risk of infection and, in the long-term, may lead to development of PTS.

The incidence of catheter related thrombosis varies among different studies, depending on the diagnostic approach, type of central lines, and patient population studied. The symptomatic DVT rates are approximately 
$2.6 \%-5 \%{ }^{48,49}$ while the rates of DVT diagnosed through imaging techniques - venogram, ultrasound, magnetic resonance venogram - reach up to $39 \% .{ }^{48}$

The risk factors for catheter related thrombosis in children are not well defined. Percutaneous inserted catheters have a higher DVT rate than surgically inserted ones, according to several adult and pediatric studies, ${ }^{50}$ though other studies did not show any significant difference. ${ }^{49}$ Femoral catheters seem to have a higher incidence of DVT in infants compared to other locations. ${ }^{50}$ Larger catheters seem to increase the risk of DVT compared to smaller ones. ${ }^{51}$ The duration of catheter use showed a correlation with the incidence of thrombosis in some studies ${ }^{52}$ but not in others. ${ }^{53,54}$ Children with complex comorbid conditions are more likely to develop catheter related DVT. ${ }^{7,50}$ The role of associated treatments like L-asparaginase or inherited thrombophilic traits is not well defined.

The treatment includes removal of the catheter, when possible, either immediately or after 4-5 days of anticoagulant therapy. If this is not possible due to the need for vascular access, the ACCP guideline ${ }^{22}$ suggest anticoagulation in the therapeutic range for 3 months followed by anticoagulation in the prophylactic range until the catheter is removed. The thrombus should be monitored with imaging studies at periodic intervals. The risk of vascular obstruction and embolism should always be balanced against the risk of bleeding with anticoagulation.

Several prophylactic interventions have been used in order to reduce the risk of catheter related DVT including heparin bonded catheters, heparin infusions, oral warfarin, and AT concentrates, none of which reduced the thrombosis risk in a convincing manner. ${ }^{55}$ The ACCP guidelines suggest no chemoprophylaxis due to paucity of data and risk of bleeding. One notable exception is the central venous lines placed in newborns which should be flushed with continuous low-dose unfractionated heparin infusion $(0.5 \mathrm{U} / \mathrm{kg} / \mathrm{hr})$.

\section{Cancer}

The incidence of symptomatic DVT in children with cancer ranges from $2 \%$ to $16 \%$ depending on the population studied and diagnostic approach. Asymptomatic DVT occurs in up to $40 \%$ of children with cancer. The DVT is located primarily in the upper and lower extremities. The incidence of PE varies between $2 \%$ and $20 \% .{ }^{56}$ The main risk factors include the presence of a central venous line - in up to $75 \%$ of cases - and the use of certain chemotherapy agents - L-asparaginase in acute lymphoblastic leukemia treatment. Older patients, patients with leukemia and sarcomas, the presence of an intra-thoracic mass, and inherited thrombophilic traits seem to increase the risk of DVT. Children with acute lymphoblastic leukemia have an increased risk of cerebral venous thrombosis. ${ }^{56}$

There are no guidelines for DVT prophylaxis in this group of patients. Several interventions have been evaluated in small studies: LMWH, ${ }^{57}$ AT supplementation, ${ }^{58}$ cryoprecipitate/fresh frozen plasma supplementation, ${ }^{59}$ and low-dose warfarin. ${ }^{60}$ None of them showed a clear benefit in reducing the incidence of DVT. ${ }^{61}$ The addition of LMWH to AT supplements was superior to AT supplementation alone in preventing DVT in all patients on asparaginase therapy. ${ }^{62}$

\section{Conclusion}

VTE has become more and more common in the pediatric population, primarily as a result of increased complexity of medical treatments. The majority of pediatric VTEs has an identifiable cause, with central venous catheters being the single most important risk factor.

Many of the treatment decisions are based on data from adult studies, though these results cannot always be extrapolated to children, especially newborns, due to age-related particularities of hemostasis.

As newer therapies, including various methods of thrombolysis, and new oral anticoagulants permeate in pediatric practice, larger, multicenter studies are needed to define their risks and benefits.

The use of central venous catheters has steadily increased over the past decades. The factors that lead to catheter related thrombosis as well as the interventions required to reduce this complication are still a matter of debate. Larger studies are needed to determine the best interventions for reducing the risk of catheter related thrombosis.

\section{Disclosure}

The author has no conflicts of interest to disclose.

\section{References}

1. Andrew M, David M, Adams M, et al. Venous thromboembolic complications (VTE) in children: first analysis of the Canadian Registry of VTE. Blood. 1994;83(5):1251-1257.

2. van Ommen CH, Heijboer H, Büller HR, Hirasing RA, Heijmans HS, Peters M. Venous thromboembolism in childhood: a prospective two-year registry in The Netherlands. J Pediatr. 2001;139(5):676-681.

3. Tuckuviene R, Christensen AL, Helgstad J, Johensen S, Kirstensen S. Pediatric Venous and Arterial Noncerbral Thromboembolism in Denmark: A Nationwide Population-Based Study. J Pediatr. 2011; 159(4):663-669.

4. Spencer FA, Emery C, Lessard D, et al. The Worcester Venous Thromboembolism Study: a population-based study of the clinical epidemiology of venous thromboembolism. J Gen Intern Med. 2006; 21(7):722-727. 
5. White RH. The epidemiology of venous thromboembolism. Circulation 2003;107(23 Suppl 1):14-18.

6. Boulet S, Grosse SD, Thornburg CD, Yusuf H, Tsai J, Hooper WC. Trends in Venous Thromboembolism-Related Hospitalizations, 1994-2009. Pediatrics. 2012;130(4):e812-e820.

7. Raffini L, Huang YS, Witmer C, Feudtner C. Dramatic increase in venous thromboembolism in children's hospitals in the United States from 2001 to 2007. Pediatrics. 2009;124(4):1001-1008.

8. Stokes S, Breheny P, Radulescu A, Radulescu VC. Impact of obesity on the risk of venous thromboembolism in an inpatient pediatric population. Pediatr Hematol Oncol. 2014;31(5):475-480.

9. Jaffray J, Young G. Developmental hemostasis: clinical implications from the fetus to the adolescent. Pediatr Clin North Am. 2013;60(6): 1407-1417.

10. Yang JY, Chan AK. Pediatric thrombophilia. Pediatr Clin North Am. 2013;60(6):1443-1462.

11. van Geffen M, van Heerde WL. Global haemostasis assays, from bench to bedside. Thromb Res. 2012;129(6):681-687.

12. Johnson NV, Khor B, Van Cott EM. Advances in laboratory testing for thrombophilia. Am J Hematol. 2012;87 Suppl 1:S108-S112.

13. Raffini L. Thrombophilia in children: who to test, how, when, and why? Hematology Am Soc Hematol Educ Program. 2008:228-235.

14. Sosothikul D, Seksarn P, Lusher JM. Pediatric reference values for molecular markers in hemostasis. J Pediatr Hematol Oncol. 2007;29(1): 19-22.

15. Biss TT, Brandão LR, Kahr WH, Chan AK, Williams S. Clinical probability score and D-dimer estimation lack utility in the diagnosis of childhood pulmonary embolism. J Thromb Haemost. 2009;7(10):1633-1638.

16. Lee EY, Tse SK, Zurakowski D, et al. Children suspected of having pulmonary embolism: multidetector CT pulmonary angiography thromboembolic risk factors and implications for appropriate use. Radiology. 2012;262(1):242-251.

17. Goldenberg NA, Knapp-Clevenger R, Manco-Johnson MJ; Mountain States Regional Thrombophilia Group. Elevated plasma factor VIII and D-dimer levels as predictors of poor outcomes of thrombosis in children. N Engl J Med. 2004;351(11):1081-1088. Erratum in: $N$ Engl J Med. 2005;352(20):2146.

18. Gokce M, Altan I, Unal S, et al. Recurrent pediatric thrombosis: the effect of underlying and/or coexisting factors. Blood Coagul Fibrinolysis. 2012;23(5):434-439.

19. Dandoy CE, Kukreja KU, Gruppo RA, Patel MN, Tarango C. Outcomes in children with deep vein thrombosis managed with percutaneous endovascular thrombolysis. Pediatr Radiol. 2015;45(5):719-726.

20. Greene LA, Goldenberg NA. Deep vein thrombosis: thrombolysis in the pediatric population. Semin Intervent Radiol. 2012;29(1):36-43.

21. Manco-Johnson MJ. How I treat venous thrombosis in children. Blood. 2006;107(1):21-29.

22. Monagle P, Chan AK, Goldenberg NA, et al. Antithrombotic Therapy and Prevention of Thrombosis, 9th ed: American College of Chest Physicians Evidence-Based Clinical Practice Guidelines. Chest. 2012; 141(2 Suppl):e737S-e801S.

23. Kuhle S, Eulemeskekian P, Kavanagh B, Massicotte P, Vegh P, Mitchell LG. A clinical significant incidence of bleeding in critically ill children receiving therapeutic doses of unfractionated heparin: a prospective cohort study. Haematologica. 2007;92(2):244-247.

24. Risch L, Fischer JE, Herklotz R, Huber AR. Heparin-induced thrombocytopenia in paediatrics: clinical characteristics, therapy and outcomes. Intensive Care Med. 2004;30(8):1615-1624.

25. Klenner AF, Lubenow N, Raschke R, Greinacher A. Heparin-induced thrombocytopenia in children: 12 new cases and review of the literature. Thromb Haemost. 2004;91(4):719-724.

26. Anderson DR, Harrison L, Hirsh J. Evaluation of a portable prothrombin time monitor for home use by patients who require long-term oral anticoagulant therapy. Arch Intern Med. 1993;153(12):1441-1447.

27. Giglia TM, Massicotte MP, Tweddell JS, et al. Prevention and treatment of thrombosis in pediatric and congenital heart disease: a scientific statement from the American Heart Association. Circulation. 2013;128(24): 2622-2703.
28. Newall F, Campbell J, Savoia H, et al. Incidence of major bleeding in a large paediatric cohort of patients requiring warfarin therapy. JThromb Haemost. 2005;3(Supplement 1):OR357.

29. Massicotte P, Julian JA, Gent M, et al. An open-label randomized controlled trial of low molecular weight heparin compared to heparin and coumadin for the treatment of venous thromboembolic events in children: the REVIVE trial. Thromb Res. 2003;109(2-3): 85-92.

30. Monagle P, Michelson AD, Bovill E, Andrew M. Antithrombotic therapy in children. Chest. 2001;119(1 Suppl):344S-370S.

31. Young G, Boshkov LK, Sullivan JE, et al. Argatroban therapy in pediatric patients requiring nonheparin anticoagulation: an open-label, safety, efficacy, and pharmacokinetic study. Pediatr Blood Cancer. 2011;56(7): 1103-1109.

32. Lyle CA, Sidonio RF, Goldenberg NA. New developments in pediatric venous thromboembolism and anticoagulation, including the target-specific oral anticoagulants. Curr Opin Pediatr. 2015;27(1): $18-25$.

33. Ko RH, Michieli C, Lira JL, Young G. FondaKIDS II: long-term followup data of children receiving fondaparinux for treatment of venous thromboembolic events. Thromb Res. 2014;134(3):643-647.

34. O'Brien SH, Kulkarni R, Wallace A, Hamblin F, Burr S, Goldenberg NA. Multicenter dose-finding and efficacy and safety outcomes in neonates and children treated with dalteparin for acute venous thromboembolism. J Thromb Haemost. 2014;12(11):1822-1825.

35. Wang M, Hays T, Balasa V, et al. Low-dose tissue plasminogen activator thrombolysis in children. J Pediatr Hematol Oncol. 2003;25(5): 379-386.

36. Goldenberg NA, Durham JD, Knapp-Clevenger R, Manco-Johnson MJ. A thrombolytic regimen for high-risk deep venous thrombosis may substantially reduce the risk of postthrombotic syndrome in children. Blood. 2007;110(1):45-53.

37. Kukreja KU, Lungren MP, Patel MN, et al. Endovascular venous thrombolysis in children younger than 24 months. JVasc Interv Radiol. 2014;25(8):1158-1164.

38. Manco-Johnson MJ, Grabowski EF, Hellgreen M, et al. Recommendations for tPA thrombolysis in children. On behalf of the Scientific Subcommittee on Perinatal and Pediatric Thrombosis of the Scientific and Standardization Committee of the International Society of Thrombosis and Haemostasis. Thromb Haemost. 2002;88(1):157-158.

39. Albisetti M. Thrombolytic therapy in children. Thromb Res. 2006; 118(1):95-105.

40. Raffini L, Cahill AM, Hellinger J, Manno C. A prospective observational study of IVC filters in pediatric patients. Pediatr Blood Cancer. 2008;51(4):517-520.

41. Kukreja KU, Gollamudi J, Patel MN, Johnson ND, Racadio JM. Inferior vena cava filters in children: our experience and suggested guidelines. J Pediatr Hematol Oncol. 2011;33(5):334-338.

42. Blevins E, Raffini L. Extensive Thrombotic Complications After Inferior Vena Cava Filter Placement in 2 Adolescents With Spinal Cord Injury: A Cautionary Tale and Review. J Pediatr Hematol Oncol. 2015;37(4):e227-e229.

43. Stem J, Christensen A, Davis D, Raffini L. Safety of prophylactic anticoagulation at a pediatric hospital. $J$ Pediatr Hematol Oncol. 2013;35(7):e287-e291.

44. Faustino EV, Hanson S, Spinella PC, et al. A multinational study of thromboprophylaxis practice in critically ill children. Crit Care Med. 2014;42(5):1232-1240.

45. Chalmers E, Ganesen V, Liesner R, et al. Guideline on the investigation, management and prevention of venous thrombosis in children. Br J Haematol. 2011;154(2):196-207.

46. Brandão LR, Simpson EA, Lau KK. Neonatal renal vein thrombosis. Semin Fetal Neonatal Med. 2011;16(6):323-328.

47. Massicotte MP, Dix D, Monagle P, Adams M, Andrew M. Central venous catheter related thrombosis in children: analysis of the Canadian Registry of Venous Thromboembolic Complications. J Pediatr. 1998;133(6):770-776. 
48. Albisetti M, Kellenberger CJ, Bergsträsser E, et al. Port-a-cath-related thrombosis and postthrombotic syndrome in pediatric oncology patients. J Pediatr. 2013;163(5):1340-1346.

49. Kanin M, Young G. Incidence of thrombosis in children with tunneled centralvenous access devices versus peripherally inserted central catheters (PICCs). Thromb Res. 2013;132(5):527-530.

50. Gray BW, Gonzalez R, Warrier KS, et al. Characterization of central venous catheter-associated deep venous thrombosis in infants. J Pediatr Surg. 2012;47(6):1159-1166.

51. Farinasso L, Bertorello N, Garbarini L, et al. Risk factors of central venous lines-related thrombosis in children with acute lymphoblastic leukemia during induction therapy: a prospective study. Leukemia. 2007;21(3):552-556.

52. Hanson SJ, Punzalan RC, Christensen MA, et al. Incidence and risk factors for venous thromboembolism in critically ill children with cardiac disease. Pediatr Cardiol. 2012;33(1):103-108.

53. Beck C, Dubois J, Grignon A, Lacroix J, David M. Incidence and risk factors of catheter-related deep vein thrombosis in a pediatric intensive care unit: a prospective study. J Pediatr. 1998;133(2):237-241.

54. Male C, Julian JA, Massicotte P, Gent M, Mitchell L; PROTEKT Study Group. Significant association with location of central venous line placement and risk of venous thrombosis in children. Thromb Haemost. 2005;94(3):516-521.

55. Vidal E, Sharathkumar A, Glover J, Faustino EV. Central venous catheter-related thrombosis and thromboprophylaxis in children: a systematic review and meta-analysis. JThromb Haemost. 2014;12(7): 1096-1109.

56. Piovesan D, Attard C, Monagle P, Ignjatovic V. Epidemiology of venous thrombosis in children with cancer. Thromb Haemost. 2014;111(6): 1015-1021.
57. Massicotte P, Julian JA, Gent M, et al. An open-label randomized controlled trial of low molecular weight heparin for the prevention of central venous line-related thrombotic complications in children: the PROTEKT trial. Thromb Res. 2003;109(2-3):101-108.

58. Mitchell L, Andrew M, Hanna K, et al. Trend to efficacy and safety using antithrombin concentrate in prevention of thrombosis in children receiving 1-asparaginase for acute lymphoblastic leukemia. Results of the PAARKA study. Thromb Haemost. 2003;90(2):235-244.

59. Abbott LS, Deevska M, Fernandez CV, et al. The impact of prophylactic fresh-frozen plasma and cryoprecipitate on the incidence of central nervous system thrombosis and hemorrhage in children with acute lymphoblastic leukemia receiving asparaginase. Blood. 2009;114(25):5146-5151.

60. Ruud E, Holmstrøm H, De Lange C, Hogstad EM, Wesenberg F. Lowdose warfarin for the prevention of central line-associated thromboses in children with malignancies - a randomized, controlled study. Acta Paediatr. 2006;95(9):1053-1059.

61. Schoot RA, Kremer LC, van de Wetering MD, van Ommen CH. Systemic treatments for the prevention of venous thrombo-embolic events in paediatric cancer patients with tunnelled central venous catheters. Cochrane Database Syst Rev. 201311;9:CD009160.

62. Meister B, Kropshofer G, Klein-Franke A, Strasak AM, Hager J, Streif W. Comparison of low-molecular-weight heparin and antithrombin versus antithrombin alone for the prevention of symptomatic venous thromboembolism in children with acute lymphoblastic leukemia. Pediatr Blood Cancer. 2008;50(2):298-303.
Pediatric Health, Medicine and Therapeutics

\section{Publish your work in this journal}

Pediatric Health, Medicine and Therapeutics is an international, peerreviewed, open access journal publishing original research, reports, editorials, reviews and commentaries. All aspects of health maintenance, preventative measures and disease treatment interventions are addressed within the journal. Practitioners from all disciplines are invited to submit

\section{Dovepress}

their work as well as healthcare researchers and patient support groups. The manuscript management system is completely online and includes a very quick and fair peer-review system. Visit http://www.dovepress.com/ testimonials.php to read real quotes from published authors. 